\title{
hnRNP E1 at the crossroads of translational regulation of epithelial-mesenchymal transition
}

\author{
Simon Grelet ${ }^{1,2}$, Philip H. Howe ${ }^{1,2}$ \\ ${ }^{1}$ Hollings Cancer Center, Medical University of South Carolina, Charleston, South Carolina 29425, USA. \\ ${ }^{2}$ Department of Biochemistry, Medical University of South Carolina, Charleston, South Carolina 29425, USA.
}

Correspondence to: Dr. Simon Grelet and Prof. Philip H. Howe, Department of Biochemistry, Medical University of South Carolina, Charleston, South Carolina 29425, USA. E-mail: grelet@musc.edu; howep@musc.edu

How to cite this article: Grelet S, Howe PH. hnRNP E1 at the crossroads of translational regulation of epithelial-mesenchymal transition. J Cancer Metastasis Treat2019;5:16. http://dx.doi.org/10.20517/2394-4722.2018.85

Received: 1 Dec 2018 First Decision: 30 Dec 2018 Revised: 10 Jan 2019 Accepted: 22 Jan 2019 Published: 11 Mar 2019

Science Editor: William P. Schiemann Copy Editor: Cai-Hong Wang Production Editor: Huan-Liang Wu

\begin{abstract}
The epithelial-mesenchymal transition (EMT), in which cells undergo a switch from a polarized, epithelial phenotype to a highly motile fibroblastic or mesenchymal phenotype is fundamental during embryonic development and can be reactivated in a variety of diseases including cancer. Spatio-temporally-regulated mechanisms are constantly orchestrated to allow cells to adapt to their constantly changing environments when disseminating to distant organs. Although numerous transcriptional regulatory factors are currently wellcharacterized, the post-transcriptional control of EMT requires continued investigation. The hnRNP E1 protein displays a major role in the control of tumor cell plasticity by regulating the translatome through multiple nonredundant mechanisms, and this role is exemplified when E1 is absent. hnRNP E1 binding to RNA molecules leads to direct or indirect translational regulation of specific sets of proteins: (1) hnRNP E1 binding to specific targets has a direct role in translation by preventing elongation of translation; (2) hnRNP E1-dependent alternative splicing can prevent the generation of a competing long non-coding RNA that acts as a decoy for microRNAs (miRNAs) involved in translational inhibition of EMT master regulators; (3) hnRNP E1 binding to the 3' untranslated region of transcripts can also positively regulate the stability of certain mRNAs to improve their translation. Globally, hnRNP E1 appears to control proteome reprogramming during cell plasticity, either by direct or indirect regulation of protein translation.
\end{abstract}

Keywords: Breast cancer, tumor progression, epithelial-mesenchymal transition, cancer stem cells, transforming growth factor- $\beta$, translation, hnRNP E1, PCBP1

\footnotetext{
(a) (1)

(C) The Author(s) 2019. Open Access This article is licensed under a Creative Commons Attribution 4.0 International License (https://creativecommons.org/licenses/by/4.0/), which permits unrestricted use, sharing, adaptation, distribution and reproduction in any medium or format, for any purpose, even commercially, as long as you give appropriate credit to the original author(s) and the source, provide a link to the Creative Commons license, and indicate if changes were made.
} 


\section{INTRODUCTION}

\section{Epithelial-mesenchymal transition in tumor progression and metastasis}

Metastasis represents a critical step in tumor progression and accounts for more than $90 \%$ of cancer-induced mortality ${ }^{[1]}$. Despite the tremendous efforts made by the scientific community over recent decades, the cellular and molecular events that control tumor cell plasticity remain incompletely elucidated.

It has been shown that the epithelial-mesenchymal transition (EMT) is essential in embryonic development and in tumor metastasis and is among the mechanisms deemed critical in tumor cell plasticity. EMT consists of a fine-tuned phenotypic switch, characterized by the loss of apical-basal polarity and cellular adhesion in epithelial cells ${ }^{[2,3]}$. Cells undergoing transition gradually express mesenchymal features, such as enhanced cytoskeletal rearrangement and extracellular matrix (ECM) degradation, both essential for cell motility [Figure 1].

EMT is not a unidirectional mechanism. The transition is a fine-tuned, reversible mechanism that allows cells to switch between epithelial and mesenchymal phenotypes while manifesting all intermediate phenotypic shades ${ }^{[2,4]}$. The reverse mechanism, known as mesenchymal-epithelial transition (MET), allows reversion to the differentiated phenotype. Reversion is important for the potential formation of metastases that can occur as tumor cells attempt to relocate to distant organs to develop secondary tumors. Due to its transience, its presence in multiple-states, and its reversible nature, EMT is technically challenging to observe throughout tumor progression in vivo ${ }^{[4]}$. Nevertheless, it is clearly demonstrated that transitioned cells harbor higher invasive capacities ${ }^{[5-7]}$. In the early steps of metastasis, epithelial cancer cells must acquire the ability to separate from the primary tumor ${ }^{[4,8]}$. Such departure may occur as single cells or as clusters of cells, and always requires the loss or the alteration of cell-to-cell and cell-to-matrix interactions ${ }^{[9]}$.

In the current model, EMT-positive tumor cells displaying newly-acquired mesenchymal features invade their surrounding environment and intravasate into the circulatory system. Cancer dissemination in this model results from ECM degradation and increased motility ${ }^{[1,11]}$. It was also proposed that the survival of circulating tumor cells (CTCs) in the blood stream was enhanced by the phenotypic plasticity observed during EMT $^{[12-15]}$. Following dissemination into the circulation, CTCs extravasate and colonize distant organs to ultimately form secondary tumors. The ultimate metastatic colonization occurs through the reepithelialization of cells by MET and is followed either by a proliferative cycle with subsequent drug resistant secondary tumor growth, or by a dormant cycle with latency of tumor relapse. Although there are countless reports demonstrating that genetic mutations are recurrently arising in many types of primary tumors, the attempts to identify genes that are recurrently mutated in the genomes of metastasized cells have consistently failed until now. Such observations are advocating for the prime role of cell plasticity in tumor cells dissemination. However, the experimental evidences of the casual function of EMT in metastasis formation remain to be clearly demonstrated. The explicit role of EMT in tumor progression remains actively debated but its implication in the increased resistance seen in both conventional and targeted antitumor therapies is currently well-accepted by the scientific community ${ }^{[12,14,16-21]}$. I seems now clear that the controversy around the role of EMT in tumor dissemination might be attributed to its non-linear and multi-modal nature and that these many intermediate stages of EMT may occupy different regions on such a multi-dimensional landscape ${ }^{[22]}$. Many groups are still investigating the role of EMT in tumor cells dissemination, and at this point, multiple hypotheses are still emerging to underline the mechanisms involved in the successful dissemination of tumor cells beyond the current EMT/MET view ${ }^{[23]}$.

\section{Heterogeneous nuclear ribonucleoprotein E1}

Heterogeneous nuclear ribonucleoproteins (hnRNPs) encompass a large family of RNA-binding proteins (RBPs) that contribute to multiple aspects of nucleic acid metabolism. These aspects include alternative splicing, mRNA stabilization, transcriptional control, and translation regulation. The coding sequences 


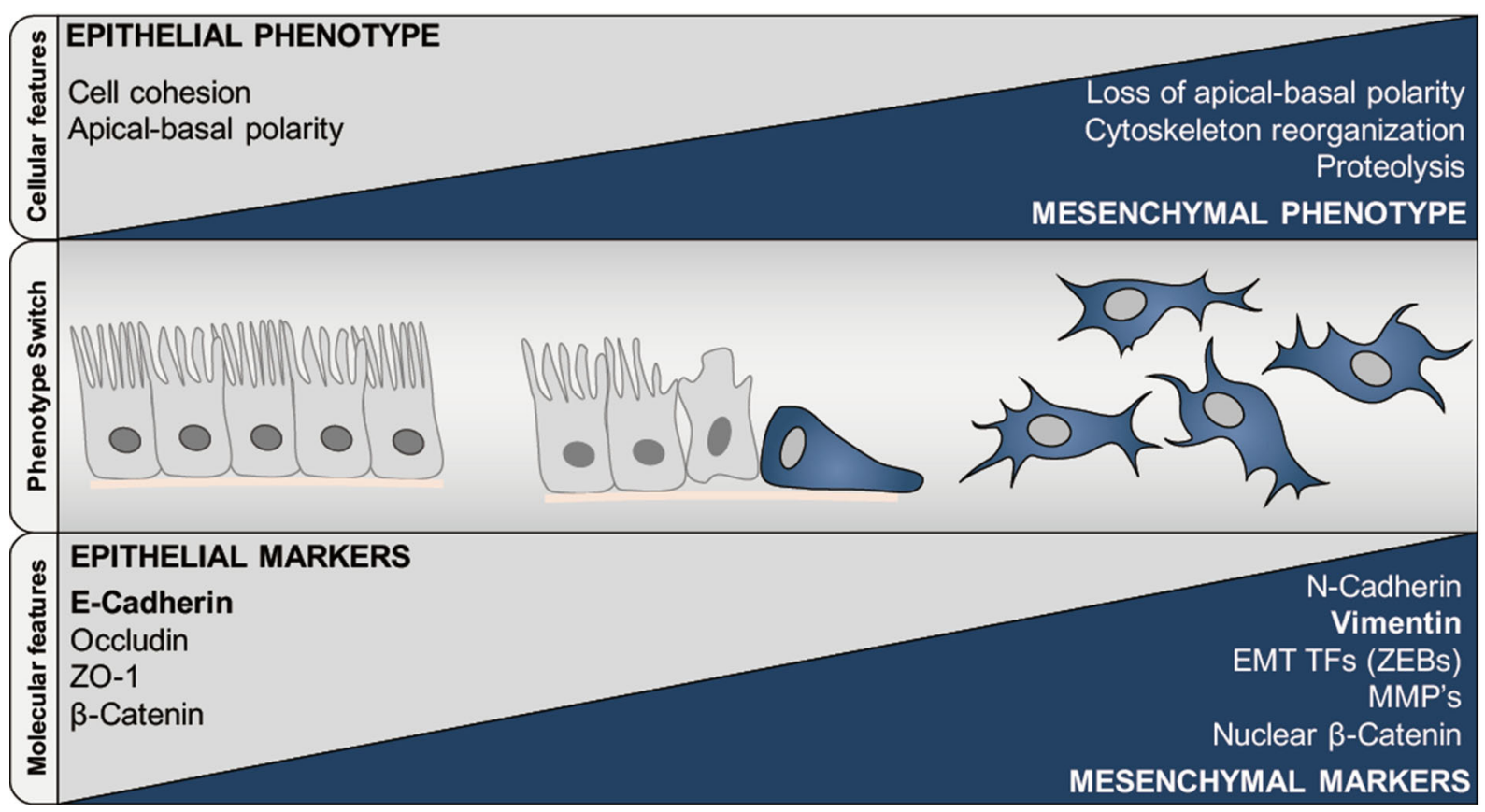

Figure 1. Epithelial-mesenchymal transition relies upon a gradually orchestrated switch from a polarized, epithelial phenotype to a highly motile fibroblastic or mesenchymal phenotype. Epithelial cells are polarized with strong cell-cell cohesions and are organized by multiple cell junction proteins such as E-Cadherin, Occludin, Zonula Occludens, $\beta$-catenin and other epithelial markers. During EMT, tumor cells lose their epithelial features and acquire a mesenchymal phenotype, which promotes their motility and invasive capacity. The switch is acquired through a deep reprogramming of the transcriptional landscape and involves activation of EMT transcription factors such as ZEBs, reorganization of cytoskeletal components by regulation of proteins such as Vimentin, and modulation of expression/secretion of invasion-mediating proteases such as matrix metalloproteinases

of hnRNPs reveal a modular structure consisting of one or more RNA-binding motifs, and at least one auxiliary domain that regulates protein-protein interactions and subcellular localization ${ }^{[24]}$. Indeed, all hnRNPs contain RNA binding domains that may include RNA recognition motifs (RRM), the quasi-RRM, glycine-rich domains and $\mathrm{KH}$ domains. Contrary to RNA-binding domains, auxiliary domains are divergent in protein sequence and are unstructured but are highly involved in regulating subcellular localization and other biological features. Most of the hnRNP proteins contain nuclear localization signals and are therefore mainly located in the nucleus during steady state. However, they can translocate into the cytosol via signaling pathway activation or by recruitment by other proteins. Importantly, for most of the hnRNPs, cellular functions are tightly regulated through post-transcriptional modifications including but not limited to methylation, phosphorylation, ubiquitination and sumoylation.

Poly(rC)-binding protein 1 (PCBP1 also called hnRNP E1) belongs to a hnRNP family that is composed of hnRNP K/J and the alpha-complex proteins (PCBP1-4 $\alpha$ or CP1-4). Proteins in this family contain three hnRNP K homology $(\mathrm{KH})$ domains for RNA-binding. The human PCBP1 gene encodes the hnRNP E1 protein, and was initially defined as clone sub2.3, with poly(C)-binding activity and observed similarity to hnRNP $\mathrm{E}^{[25]}$. hnRNP E1 harbors three highly conserved $\mathrm{KH}$ domains, $\mathrm{KH} 1$ to $\mathrm{KH} 3 . \mathrm{KH} 1$ and $\mathrm{KH} 3$ domains were initially predicted to bind to RNA and in vitro studies later showed that the $\mathrm{KH} 2$ domain of hnRNP E1 also binds to RNA to regulate protein translation ${ }^{[26-28]}$. The hnRNP E1 and hnRNP E2 proteins share $82 \%$ amino acid identity, with an even higher level of conservation (93\%) for their $\mathrm{KH}$ domains ${ }^{[24]}$.

The subcellular localization of hnRNP E1 is predominantly nuclear, and demonstrates accumulation within nuclear speckles, with precise sites of accumulation observed with splicing factors prior to nascent transcript loading ${ }^{[29]}$. The nuclear localization of hnRNP E1 is abolished by Actinomycin $\mathrm{D}^{[30]}$. Since splicing factors undergo continuous and rapid nucleo-cytoplasmic shuttling ${ }^{[31]}$, and because splicing is coupled to 
transcription, RNA polymerase inhibition results in the cytoplasmic accumulation of many splicing factors. Such observations support the primordial nuclear role of hnRNP E1 in pre-RNA splicing ${ }^{[32]}$. The subcellular localization of hnRNP E1 is also governed by cell signaling stimuli. It was demonstrated that SMAD3 and hnRNP E1 were induced to govern alternative splicing, mediated by their colocalization in SC35 (also known as SRSF2)-positive nuclear speckles, downstream of EGF and TGFb, respectively ${ }^{[33]}$. Even if it is to a lesser extent, hnRNP E1 is also clearly observed in the cytoplasm, and its presence potentially correlates to its function as a translational regulator ${ }^{[29,34,35]}$.

\section{REGULATION OF TRANSCRIPTION}

HnRNP E1 functions as a regulator of gene expression at the transcriptional level, although this does not appear to be its primary role. Recombinant cloning of the four members (PCBP1 to PCBP4) of the poly(C) binding protein family (PCBP) demonstrated that transcriptional activity of the mouse $\mu$-opioid receptor (MOR) gene increased due to interaction between PCBPs and the 26-bp polypyrimidine stretch in the MOR proximal promoter ${ }^{[36]}$. PCBPs can bind either to single-stranded or to double-stranded DNA ${ }^{[27]}$. hnRNP E1 also exhibits a mild but consistent activation of the promoter of the BRCA1 gene ${ }^{[37]}$. Finally, hnRNP E1 was found to regulate eIF4E transcription ${ }^{[38]}$. Recruitment of hnRNP E1 to the eIF4E basal element (4EBE) in the eIF4E promoter region occurs downstream of serum or EGF-mediated cellular stimulation, and this mechanism requires Pak1-dependent phosphorylation of hnRNP E1 protein ${ }^{[38]}$. These findings suggest that both hnRNP E1 and its phosphorylation downstream of growth factor-induced signaling play a regulatory role in eIF4E transcription in mitogen-stimulated cells.

\section{TRANSLATION REGULATION}

The hnRNP E1 protein regulates translation through either direct or indirect mechanisms. Examples of the most common mechanisms include regulation of mRNA stability, direct control of the ribosomal machinery, or the generation of RNA species that prevent miRNA-mediated translational repression of specific mRNAs.

\section{Control of mRNA stability}

The role of hnRNP E1 in RNA stability is exemplified by a broad spectrum of mRNA interactions. Mainly, hnRNP E1 regulates gene expression via binding to specific AU-rich elements (AREs) or U-rich elements located in the 3 ' untranslated regions (UTR) of target mRNAs. For instance, the binding of hnRNP E1 to p2 $7^{\text {kip1 }} 3^{\prime}$ UTR via its KH1 domain stabilizes p2 $7^{\text {kip1 }}$ mRNA, fueling p $27^{\text {kip1 }}$ protein expression by enhancing its translation prior to degradation. The upregulated $\mathrm{p} 27^{\text {kipl }}$ protein consequently inhibits cell proliferation, cell cycle progression, and tumorigenesis, and can concurrently promote cell apoptosis under paclitaxel treatment ${ }^{[39]}$. Interestingly, other cyclin-dependent kinase inhibitors such as $\mathrm{p}_{21}{ }^{\text {Waf1 }}$ are also regulated through hnRNP E1 mediated mRNA stability. Co-immunopurification of p21 Waf1 $\mathrm{mRNA}$ from MDAMB-468 breast cancer cells using hnRNP E1 antibody suggests that hnRNP E1 protein binds to one or more motifs distributed throughout the p21 ${ }^{\text {Waf1 }} 3$ ' UTR to stabilize the mRNA ${ }^{[30]}$.

The eNOS mRNA 3' UTR contains multiple evolutionarily conserved pyrimidine (C and CU)-rich sequence elements that are both necessary and sufficient for mRNA stabilization. The hnRNP E1 protein binds to these 3' UTR elements. Hence, hnRNP E1is recruited to a stabilizing RNP complex that protects eNOS mRNA from the inhibitory effects of its antisense transcript sONE, and from 3' UTR-targeting small interfering RNAs (siRNAs) and miRNAs ${ }^{[40]}$. HnRNP E1 regulates the stability of p63 mRNA via binding to a CU-rich element (CUE) within the p63 $3^{\prime} \mathrm{UTR}^{[4]}$. The p63 protein contribution to EMT could vary according to the biological context. It has been shown that p63 directly modulates the tumor cell plasticity by either attenuating EMT in human prostate cancer cells or promoting tumor cell invasion during human and mouse breast tumor cells dissemination ${ }^{[42,43]}$. Phosphorylation of hnRNP E1 also contributes to stabilization 
of mu opioid receptor (MOR) mRNA via interaction with ARE RNA-binding protein 1 (AUF1) and poly A binding protein $(\mathrm{PABP})^{[44]}$.

\section{Alternative polyadenylation}

The addition of the poly-(A) to the messenger RNA 3' UTR is a co-transcriptional process occurring in the nucleus. As 3' UTRs contain cis elements that are involved in various aspects of mRNA metabolism, 3' UTR alternative polyadenylation (APA) can affect post-transcriptional gene regulation considerably in various ways, including modulation of stability, translation, nuclear export and cellular localization of mRNA. 3' UTR-APA can also affect the localization of the encoded protein ${ }^{[45]}$. Polyadenylation of mRNA is a two-step process consisting of endonucleolytic cleavage and addition of an untemplated poly(A) tail.

The role for hnRNP E1 in the regulation of alternative polyadenylation has been established in an in vitrotranscribed and polyadenylated alpha-globin $3^{\prime}$ UTR assay ${ }^{[4]}$. Furthermore, a screening study for alternative polyadenylation utilizing RNA interference (RNAi) identified hnRNP E1 as the second highest factor in the control of polyadenylation signal usage. The mechanism by which hnRNP E1 modulates polyadenylation has yet to be characterized ${ }^{[47]}$.

\section{Direct control of translation machinery through BAT elements}

As we and others have previously demonstrated, the regulation of gene expression at the post-transcriptional level plays an indispensable role in TGF $\beta$-induced EMT and metastasis ${ }^{[30,34,48-52]}$. We identified a transcriptselective translational regulatory pathway in which a ribonucleoprotein (mRNP) complex binds to a 33-nucleotide TGF 3 -activated translation (BAT) element in the mRNA 3' UTR and silences the translation of a cohort of mesenchymal protein-encoding mRNAs. HnRNP E1 is a critical component of the BAT-binding mRNP complex ${ }^{[53]}$. Silenced mRNAs include Disabled2 (Dab2) and Interleukin-like EMT-inducer (ILEI), which are involved in mediating $\mathrm{EMT}^{[34,51,54,55]}$. Furthermore, TGF $\beta$ activates a kinase cascade terminating in the phosphorylation of hnRNP E1 protein at Serine 43. This phosphorylation occurs by isoform-2 specific activation of Akt and induces the release of the mRNP complex from the BAT element. This results in the reversal of translational silencing of mesenchymal protein-encoding transcripts that are required for EMT. By using a genome-wide combinatorial approach involving expression, polysome profiling and RIP-Chip analysis, we have identified the members of the cohort of translationally regulated mRNAs that are induced during TGFß-mediated $\mathrm{EMT}^{[53]}$.

At the molecular level, the eukaryotic elongation factor-1 A1 (eEF1A1) is an important additional functional component of the mRNP complex. We have previously demonstrated that the BAT element, hnRNP E1 and eEF1A1 form a ternary complex that mediates translational silencing at the translational elongation step $^{[35]}$. In non-stimulated cells exhibiting epithelial characteristics, hnRNP E1 binds to eEF1A1 and blocks progression of the $80 \mathrm{~S}$ ribosome by preventing the release of eEF1A1 from the ribosomal A-site following GTP hydrolysis. EMT induced by either TGF $\beta$ or hnRNP E1 knockdown disrupts the mRNP complex, allowing eEF1A1-mediated translational elongation of mesenchymal transcripts to proceed ${ }^{[34,35]}$ [Figure 2].

This mode of translational regulation represents an unusual case of dependency upon either agonists or stimuli to upregulate translation through 3'-UTR elements. Thus, the elucidation of this post-transcriptional regulatory pathway identified an "EMT gene signature" and provided mechanistic information as to how cell plasticity could be tightly regulated. Taken together, this work underscores the contribution of the nonphosphorylated hnRNP E1 protein to maintenance of epithelial cell integrity under normal conditions. During tumor-related epithelial plasticity, hnRNP E1 also acts as the trigger for the reversal of translational silencing, resulting in a fine-tuned, spatio-temporally controlled increase in mesenchymal protein expression. 


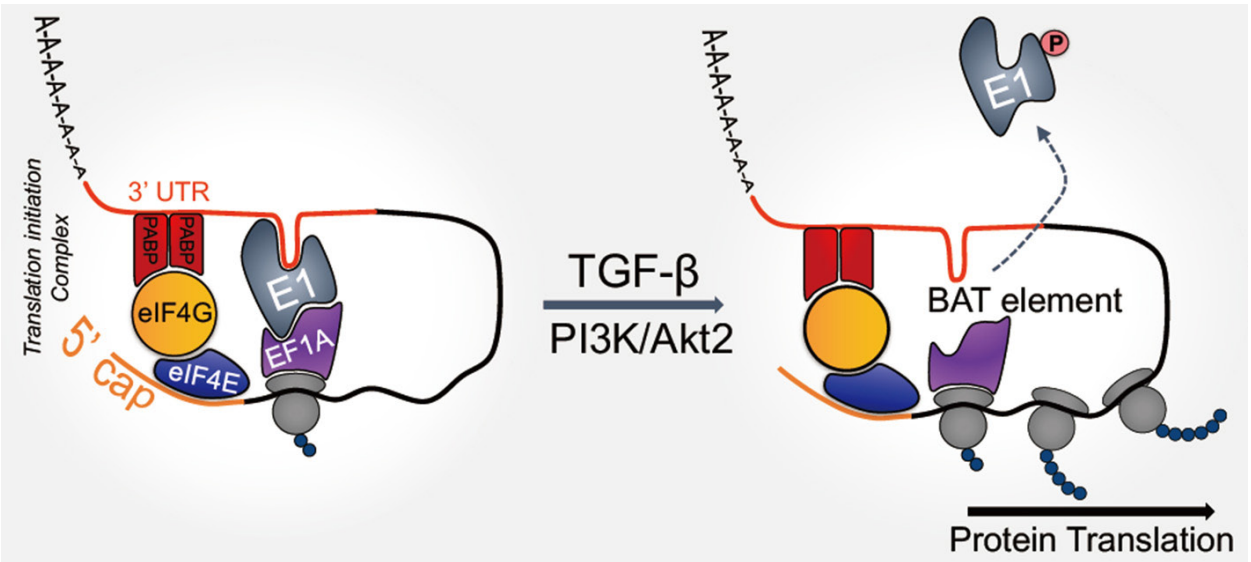

Figure 2. Molecular mechanism of hnRNP E1-mediated translational silencing. The eukaryotic elongation factor-1A1 (eEF1A1) forms a complex with hnRNP E1 and the BAT element, and silences specific protein expression by stalling the elongation of their translation by ribosomes. Given the necessity for cognate-codon interaction with the ribosomal A site, it is likely that the formation of the BAT mRNP complex occurs post-delivery of the aminoacyl-tRNA to the ribosome. The ability of the BAT mRNP complex to inhibit eEF1A1dependent elongation suggests that the $3^{\prime}$-UTR is interacting with the $5^{\prime}$-UTR in a circularized model to facilitate its proximity to the 805 ribosome ${ }^{[35]}$. It has been suggested that translatable mRNAs are likely to be found in circular forms due to interaction between PABP, elF4G, and the cap-binding protein elF4E ${ }^{[56]}$

\section{Indirect control of translation through alternative splicing}

Alternative splicing regulates over $90 \%$ of multi-exon protein-coding genes in humans ${ }^{[57]}$. Abnormal regulation of alternative splicing often produces disease-specific protein isoforms ${ }^{[58,59]}$. Additionally, genomewide analysis has identified tens of thousands of "splice variant" mRNAs that are enriched in a wide range of human diseases ${ }^{[60,61]}$. The hnRNP E1 protein is well documented for its repressive role in alternative splicing mechanisms as they apply to human health and disease. For instance, hnRNP E1 represses tumor cell invasion by inhibiting the alternative splicing of $\mathrm{CD} 44^{[62]}$. It was therefore demonstrated that enforced hnRNP E1 expression inhibited CD44 variants expression in HepG2 human liver cancer cells while knockdown of endogenous hnRNP E1 induced these variants splicing ${ }^{[62]}$. Interestingly, another study based on in vitro and in vivo models of breast cancer progression demonstrated the switch of CD44 expression occurring from variant isoforms to the standard isoform during EMT. This isoform switch to CD44s was essential for cells to undergo EMT and was required for the formation of breast tumors that display EMT characteristics in mice ${ }^{[63]}$. HnRNP E1 also binds to the growth hormone receptor pseudoexon and prevents its usage to allow the expression of a functional protein ${ }^{[6]]}$. Disruption of hnRNP E1 binding and subsequent activation of an alternative splicing event responsible for Laron syndrome was demonstrated either by hnRNP E1 knockdown or by alterations to the genomic pseudosite. We also recently reported the binding of hnRNP E1 to a pre-RNA pseudosite in the serine/threonine-protein phosphatase 1 regulatory subunit 10 (PNUTS) transcript ${ }^{[30]}$. The hnRNP E1 protein binds to a conserved BAT element that is similar in structure to those observed in the 3'-UTRs of the mesenchymal encoding mRNAs discussed above. The loss of hnRNP E1 binding to the alternative splicing site of PNUTS following hnRNP E1 knockdown, phosphorylation, and/or cytoplasmic translocation activates usage of the pseudosite and generates an alternatively spliced isoform of PNUTS. This alternative PNUTS isoform does not encode a functional protein, but rather a noncoding isoform of the gene. Functionally, the lncRNA-PNUTS acts as a decoy for miRNA-205 and binds to the miRNA causing a decrease in miRNA-205 bioavailability. This abolishes translational inhibition of mesenchymal factors such as ZEB mRNAs that would otherwise be suppressed in epithelial cells ${ }^{[30]}$. Since alternative splicing and generation of lncRNA-PNUTS is an early event in TGFß-mediated EMT, the lncRNA-PNUTS likely operates as a transient inhibitor of the miRNA-205 to allow for the temporal upregulation of ZEBs and subsequent regulation of downstream EMT events. Indeed ZEBs proteins are reciprocally linked in a feedback loop with the miR-200 family, each strictly controlling the expression of the other ${ }^{[18,65]}$. In this way, a transient, but nevertheless, strong decrease in miR-205 bioavailability, sufficient to activate the ZEB proteins, would allow for transcriptional repression of the miR-200 family or other miRNAs 


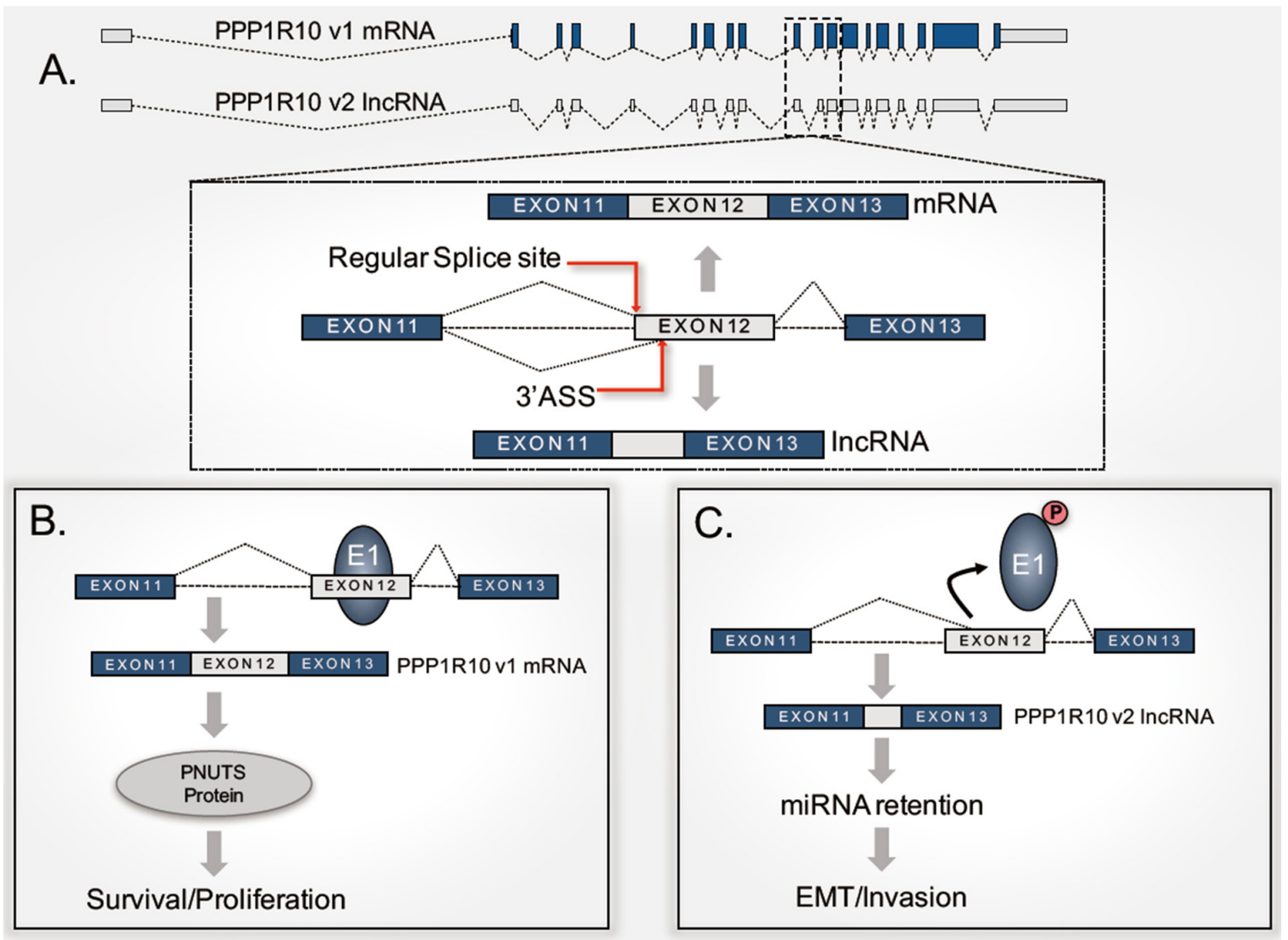

Figure 3. Molecular mechanism of hnRNP E1-mediated alternative splicing of PNUTS. A: The PPP1R10 (PNUTS) gene locus can encode either a protein coding mRNA or a non-coding RNA isoform. The PNUTS gene locus is highly conserved between human and mouse and expresses both coding and non-coding transcripts. The IncRNA-PNUTS is generated by the usage of the $3^{\prime}$ alternative splice site ( $3^{\prime} \mathrm{ASS}$ ) located at the $5^{\prime}$ end of exon 12. This usage leads to the change of the open reading frame and the generation of a premature stop codon; B: the binding of hnRNP E1 to a BAT consensus element located in the alternative splicing site results on its masking and prevents its usage to generate the PPP1R10 mRNA translated into the PNUTS protein; C: loss of hnRNP E1 binding to the alternative splice site uncovers it and allows its usage by the spliceosome machinery. The IncRNA-PNUTS acts as a decoy for miRNA-205 and thus allows the de-repression of ZEB protein translation. Reactivated expression of ZEB proteins induces the shutdown of epithelial markers such as E-Cadherin, allowing EMT to proceed

such as miR-183 or miR-203 thereby further stabilizing ZEB proteins and reinforcing the EMT process ${ }^{[30]}$. Moreover, it has been suggested that the feedback loop of miR-200/ZEB also generates hybrid phenotypes of the cells during EMT-mediated tumor cell plasticity ${ }^{[66]}$ [Figure 3].

\section{CONCLUSION}

For many years, characterization of the role of RBPs in tumor biology and cell plasticity resulted in substantial progress, and the investigation of hnRNP E1 provided understanding of many facets of its molecular function in cells. The function of the PCBP1 gene encoding the hnRNP E1 protein was first demonstrated through characterization of its role as a negative regulator of alternative splicing. Since then, many additional roles have also been discovered, and most of them appear to have critical participation in the maintenance of cell phenotype integrity ${ }^{[34,39,67]}$. At the molecular level, the ability of hnRNP E1 to specifically bind to mRNA species often leads to a direct or indirect regulation of their translation. This occurs either by controlling processivity of ribosomal machinery, stabilizing mRNA, or locking/unlocking dormant translational controls. Since it is well established that hnRNP E1 controls cell plasticity in health and disease through multiple fine-tuned regulatory mechanisms, it will be essential to develop investigations involving novel therapeutic strategies. Moreover, targeting the KH domain of hnRNP E1 may be relevant. 
However, because hnRNP-E1 is pleiotropic and ubiquitous, confining therapeutic strategies to tumor cells may be challenging.

The phenotypic changes observed during cell plasticity and tumor progression demand radical proteomic reprogramming of cells concomitant with a modulation of the codon usage by the translational machinery. Accumulation of evidence advocates for acknowledgement of a primary role for hnRNP E1 in cell plasticity, and this is reinforced by identification of mechanisms involving hnRNP E1 that directly or indirectly converge upon the translational control of plasticity-associated proteins. We therefore propose that hnRNP E1, together with its associated proteins, acts as a hub that orchestrates the demands of proteome reprogramming during health and disease-associated cell plasticity.

\section{DECLARATIONS}

\section{Acknowledgments}

We would like to thank W. Scott Streitfeld for providing helpful feedback during the preparation of this manuscript.

\section{Authors' contributions}

Wrote the manuscript: Grelet S

Validated the manuscript: Howe PH

\section{Availability of data and materials}

Not applicable.

\section{Financial support and sponsorship}

This work was supported by Hollings Cancer Center Postdoctoral Fellowship to Grelet S and the National Cancer Institute (CA154663) to Howe PH.

\section{Conflicts of interest}

Both authors declared that there are no conflicts of interest.

\section{Ethical approval and consent to participate}

Not applicable.

\section{Consent for publication}

Not applicable.

\section{Copyright}

(c) The Author(s) 2019.

\section{REFERENCES}

1. Chaffer CL, Weinberg RA. A perspective on cancer cell metastasis. Science 2011;331:1559-64.

2. Thiery JP, Acloque H, Huang RY, Nieto MA. Epithelial-mesenchymal transitions in development and disease. Cell 2009;139:871-90.

3. Nieto MA, Huang RY, Jackson RA, Thiery JP. EMT: 2016. Cell 2016;166:21-45.

4. Dongre A, Weinberg RA. New insights into the mechanisms of epithelial-mesenchymal transition and implications for cancer. Nat Rev Mol Cell Biol 2019;20:69-84.

5. Pignatelli J, Goswami S, Jones JG, Rohan TE, Pieri E, et al. Invasive breast carcinoma cells from patients exhibit MenaINV- and macrophage-dependent transendothelial migration. Sci Signal 2014;7:ra112.

6. Liu F, Gu LN, Shan BE, Geng CZ, Sang MX. Biomarkers for EMT and MET in breast cancer: an update. Oncol Lett 2016;12:4869-76.

7. Aiello NM, Maddipati R, Norgard RJ, Balli D, Li J, et al. EMT subtype Influences epithelial plasticity and mode of cell migration. Dev Cell 2018;45:681-95.e4.

8. Wang W, Goswami S, Lapidus K, Wells AL, Wyckoff JB, et al. Identification and testing of a gene expression signature of invasive 
carcinoma cells within primary mammary tumors. Cancer Res 2004;64:8585-94.

9. Friedl P, Gilmour D. Collective cell migration in morphogenesis, regeneration and cancer. Nat Rev Mol Cell Biol 2009;10:445-57.

10. Polette M, Nawrocki-Raby B, Gilles C, Clavel C, Birembaut P. Tumour invasion and matrix metalloproteinases. Crit Rev Oncol Hematol 2004;49:179-86.

11. Juncker-Jensen A, Deryugina EI, Rimann I, Zajac E, Kupriyanova TA, et al. Tumor MMP-1 activates endothelial PAR1 to facilitate vascular intravasation and metastatic dissemination. Cancer Res 2013;73:4196-211.

12. Francart ME, Lambert J, Vanwynsberghe AM, Thompson EW, Bourcy M, et al. Epithelial-mesenchymal plasticity and circulating tumor cells: travel companions to metastases. Dev Dyn Off Publ Am Assoc Anat 2018;247:432-50.

13. Bourcy M, Suarez-Carmona M, Lambert J, Francart ME, Schroeder H, et al. Tissue factor induced by epithelial-mesenchymal transition triggers a procoagulant state that drives metastasis of circulating tumor cells. Cancer Res 2016;76:4270-82.

14. Papadaki MA, Stoupis G, Theodoropoulos PA, Mavroudis D, Georgoulias V, et al. Circulating tumor cells with stemness and epithelialto-mesenchymal transition features are chemoresistant and predictive of poor outcome in metastatic breast cancer. Mol Cancer Ther 2019;18:437-47

15. Micalizzi DS, Maheswaran S, Haber DA. A conduit to metastasis: circulating tumor cell biology. Genes Dev 2017;31:1827-40.

16. Zheng X, Carstens JL, Kim J, Scheible M, Kaye J, et al. Epithelial-to-mesenchymal transition is dispensable for metastasis but induces chemoresistance in pancreatic cancer. Nature 2015;527:525-30.

17. Fischer KR, Durrans A, Lee S, Sheng J, Li F, et al. Epithelial-to-mesenchymal transition is not required for lung metastasis but contributes to chemoresistance. Nature 2015;527:472-6.

18. Krebs AM, Mitschke J, Lasierra Losada M, Schmalhofer O, Boerries M, et al. The EMT-activator Zeb1 is a key factor for cell plasticity and promotes metastasis in pancreatic cancer. Nat Cell Biol 2017;19:518-29.

19. Aiello NM, Brabletz T, Kang Y, Nieto MA, Weinberg RA, et al. Upholding a role for EMT in pancreatic cancer metastasis. Nature 2017;547:E7-8.

20. Ye X, Brabletz T, Kang Y, Longmore GD, Nieto MA, et al. Upholding a role for EMT in breast cancer metastasis. Nature 2017;547:E1-3.

21. Brabletz T, Kalluri R, Nieto MA, Weinberg RA. EMT in cancer. Nat Rev Cancer 2018;18:128-34.

22. Saunders LR, McClay DR. Sub-circuits of a gene regulatory network control a developmental epithelial-mesenchymal transition. Dev Camb Engl 2014;141:1503-13.

23. Jolly MK, Ware KE, Gilja S, Somarelli JA, Levine H. EMT and MET: necessary or permissive for metastasis? Mol Oncol 2017;11:755-69.

24. Chaudhury A, Chander P, Howe PH. Heterogeneous nuclear ribonucleoproteins (hnRNPs) in cellular processes: focus on hnRNP E1's multifunctional regulatory roles. RNA 2010;16:1449-62.

25. Aasheim HC, Loukianova T, Deggerdal A, Smeland EB. Tissue specific expression and cDNA structure of a human transcript encoding a nucleic acid binding [oligo(dC)] protein related to the pre-mRNA binding protein K. Nucleic Acids Res 1994;22:959-64.

26. Leffers H, Dejgaard K, Celis JE. Characterisation of two major cellular poly(rC)-binding human proteins, each containing three K-homologous (KH) domains. Eur J Biochem 1995;230:447-53.

27. Dejgaard K, Leffers H. Characterisation of the nucleic-acid-binding activity of KH domains. Different properties of different domains. Eur J Biochem 1996;241:425-31.

28. Brown AS, Mohanty BK, Howe PH. Identification and characterization of an hnRNP E1 translational silencing motif. Nucleic Acids Res 2016;44:5892-907.

29. Chkheidze AN, Liebhaber SA. A novel set of nuclear localization signals determine distributions of the alphaCP RNA-binding proteins. Mol Cell Biol 2003;23:8405-15.

30. Grelet S, Link LA, Howley B, Obellianne C, Palanisamy V, et al. A regulated PNUTS mRNA to lncRNA splice switch mediates EMT and tumour progression. Nat Cell Biol 2017;19:1105-15.

31. van der Houven van Oordt W, Diaz-Meco MT, Lozano J, Krainer AR, Moscat J, et al. The MKK(3/6)-p38-signaling cascade alters the subcellular distribution of hnRNP A1 and modulates alternative splicing regulation. J Cell Biol 2000;149:307-16.

32. Tennyson CN, Klamut HJ, Worton RG. The human dystrophin gene requires 16 hours to be transcribed and is cotranscriptionally spliced. Nat Genet 1995;9:184-90.

33. Tripathi V, Sixt KM, Gao S, Xu X, Huang J, et al. Direct regulation of alternative splicing by SMAD3 through PCBP1 is essential to the tumor-promoting role of TGF- $\beta$. Mol Cell 2016;64:549-64.

34. Chaudhury A, Hussey GS, Ray PS, Jin G, Fox PL, et al. TGF-beta-mediated phosphorylation of hnRNP E1 induces EMT via transcriptselective translational induction of Dab2 and ILEI. Nat Cell Biol 2010;12:286-93.

35. Hussey GS, Chaudhury A, Dawson AE, Lindner DJ, Knudsen CR, et al. Identification of an mRNP complex regulating tumorigenesis at the translational elongation step. Mol Cell 2011;41:419-31

36. Kim SS, Pandey KK, Choi HS, Kim SY, Law PY, et al. Poly(C) binding protein family is a transcription factor in $\mu$-opioid receptor gene expression. Mol Pharmacol 2005;68:729-36.

37. Thakur S, Nakamura T, Calin G, Russo A, Tamburrino JF, et al. Regulation of BRCA1 transcription by specific single-stranded DNA binding factors. Mol Cell Biol 2003;23:3774-87.

38. Meng Q, Rayala SK, Gururaj AE, Talukder AH, O'Malley BW, et al. Signaling-dependent and coordinated regulation of transcription, splicing, and translation resides in a single coregulator, PCBP1. Proc Natl Acad Sci U S A 2007;104:5866-71.

39. Shi H, Li H, Yuan R, Guan W, Zhang X, et al. PCBP1 depletion promotes tumorigenesis through attenuation of p27Kip1 mRNA stability and translation. J Exp Clin Cancer Res 2018;37:187.

40. Ho JJ, Robb GB, Tai SC, Turgeon PJ, Mawji IA, et al. Active stabilization of human endothelial nitric oxide synthase mRNA by hnRNP E1 protects against antisense RNA and microRNAs. Mol Cell Biol 2013;33:2029-46.

41. Cho SJ, Jung YS, Chen X. Poly (C)-binding protein 1 regulates p63 expression through mRNA stability. PLoS One 2013;8:e71724.

42. Jolly MK, Boareto M, Debeb BG, Aceto N, Farach-Carson MC, et al. Inflammatory breast cancer: a model for investigating cluster-based 
dissemination. NPJ Breast Cancer 2017;3:21.

43. Olsen JR, Oyan AM, Rostad K, Hellem MR, Liu J, et al. p63 attenuates epithelial to mesenchymal potential in an experimental prostate cell model. PLoS One 2013;8:e62547.

44. Hwang CK, Wagley Y, Law PY, Wei LN, Loh HH. Phosphorylation of poly(rC) binding protein 1 (PCBP1) contributes to stabilization of mu opioid receptor (MOR) mRNA via interaction with AU-rich element RNA-binding protein 1 (AUF1) and poly A binding protein (PABP). Gene 2017;598:113-30.

45. Tian B, Manley JL. Alternative polyadenylation of mRNA precursors. Nat Rev Mol Cell Biol 2017;18:18-30.

46. Wang Z, Day N, Trifillis P, Kiledjian M. An mRNA stability complex functions with Poly(A)-binding protein to stabilize mRNA in vitro. Mol Cell Biol 1999;19:4552-60.

47. Jenal M, Elkon R, Loayza-Puch F, van Haaften G, Kühn U, et al. The Poly(A)-binding protein nuclear 1 suppresses alternative cleavage and polyadenylation sites. Cell 2012;149:538-53.

48. Grelet S, Andries V, Polette M, Gilles C, Staes K, et al. The human NANOS3 gene contributes to lung tumour invasion by inducing epithelial-mesenchymal transition. J Pathol 2015;23:25-37.

49. Grelet S, McShane A, Geslain R, Howe PH. Pleiotropic roles of non-coding RNAs in TGF- $\beta$-mediated epithelial-mesenchymal transition and their functions in tumor progression. Cancers 2017;9:E75.

50. Wang H, Vardy LA, Tan CP, Loo JM, Guo K, et al. PCBP1 suppresses the translation of metastasis-associated PRL-3 phosphatase. Cancer Cell 2010;18:52-62.

51. Waerner T, Alacakaptan M, Tamir I, Oberauer R, Gal A, et al. ILEI: a cytokine essential for EMT, tumor formation, and late events in metastasis in epithelial cells. Cancer Cell 2006;10:227-39.

52. Cursons J, Pillman KA, Scheer KG, Gregory PA, Foroutan M, et al. Combinatorial targeting by MicroRNAs Co-ordinates posttranscriptional control of EMT. Cell Syst 2018;7:77-91.e7.

53. Hussey GS, Link LA, Brown AS, Howley BV, Chaudhury A, et al. Establishment of a TGF $\beta$-induced post-transcriptional EMT gene signature. PLoS One 2012;7:e52624.

54. Prunier C, Howe PH. Disabled-2 (Dab2) is required for transforming growth factor beta-induced epithelial to mesenchymal transition (EMT). J Biol Chem 2005;280:17540-8.

55. Song Q, Sheng W, Zhang X, Jiao S, Li F. ILEI drives epithelial to mesenchymal transition and metastatic progression in the lung cancer cell line A549. Tumour Biol 2014;35:1377-82.

56. Wells SE, Hillner PE, Vale RD, Sachs AB. Circularization of mRNA by eukaryotic translation initiation factors. Mol Cell 1998;2:135-40.

57. Wang ET, Sandberg R, Luo S, Khrebtukova I, Zhang L, et al. Alternative isoform regulation in human tissue transcriptomes. Nature 2008;456:470-6.

58. Paronetto MP, Passacantilli I, Sette C. Alternative splicing and cell survival: from tissue homeostasis to disease. Cell Death Differ 2016;23:1919-29.

59. Singh RK, Cooper TA. Pre-mRNA splicing in disease and therapeutics. Trends Mol Med 2012;18:472-82.

60. Song X, Zeng Z, Wei H, Wang Z. Alternative splicing in cancers: from aberrant regulation to new therapeutics. Semin Cell Dev Biol 2018;75:13-22.

61. Xiong HY, Alipanahi B, Lee LJ, Bretschneider H, Merico D, et al. RNA splicing. The human splicing code reveals new insights into the genetic determinants of disease. Science 2015;347:1254806.

62. Zhang T, Huang XH, Dong L, Hu D, Ge C, et al. PCBP-1 regulates alternative splicing of the CD44 gene and inhibits invasion in human hepatoma cell line HepG2 cells. Mol Cancer 2010;9:72.

63. Brown RL, Reinke LM, Damerow MS, Perez D, Chodosh LA, et al. CD44 splice isoform switching in human and mouse epithelium is essential for epithelial-mesenchymal transition and breast cancer progression. J Clin Invest 2011;121:1064-74.

64. Akker SA, Misra S, Aslam S, Morgan EL, Smith PJ, et al. Pre-spliceosomal binding of U1 small nuclear ribonucleoprotein (RNP) and heterogenous nuclear RNP E1 is associated with suppression of a growth hormone receptor pseudoexon. Mol Endocrinol Baltim Md 2007;21:2529-40.

65. Brabletz S, Brabletz T. The ZEB/miR-200 feedback loop - a motor of cellular plasticity in development and cancer? EMBO Rep 2010;11:670-7.

66. Lu M, Jolly MK, Levine H, Onuchic JN, Ben-Jacob E. MicroRNA-based regulation of epithelial-hybrid-mesenchymal fate determination. Proc Natl Acad Sci U S A 2013;110:18144-9.

67. Link LA, Howley BV, Hussey GS, Howe PH. PCBP1/HNRNP E1 protects chromosomal integrity by translational regulation of CDC27. Mol Cancer Res 2016;14:634-46. 